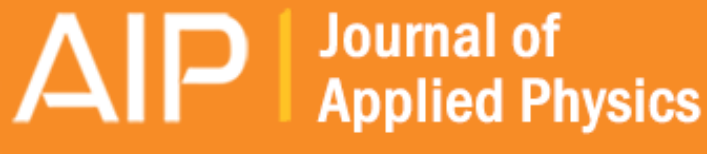

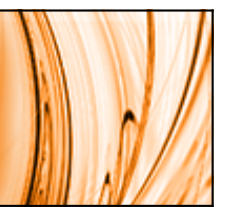

\section{Trends of hybridization-induced magnetism in cerium monochalcogenides}

N. Kioussis, D. Swearingen, B. R. Cooper, and J. M. Wills

Citation: Journal of Applied Physics 69, 5475 (1991); doi: 10.1063/1.347970

View online: http://dx.doi.org/10.1063/1.347970

View Table of Contents: http://scitation.aip.org/content/aip/journal/jap/69/8?ver=pdfcov

Published by the AIP Publishing

\section{Articles you may be interested in}

Hybridization-induced magnetism in correlated cerium systems

J. Appl. Phys. 79, 6420 (1996); 10.1063/1.361957

Re-entrant magnetic behavior of disordered alloy

AIP Conf. Proc. 286, 303 (1992); 10.1063/1.44701

Electronic structure and magnetic properties of Co-rich ternary compounds: YCo10V2

J. Appl. Phys. 69, 5703 (1991); 10.1063/1.347893

Synthesis of band and model Hamiltonian theory for strongly hybridizing uranium systems

J. Appl. Phys. 67, 5197 (1990); 10.1063/1.344660

Absolute evaluation of magnetic ordering in correlated f-electron systems

J. Appl. Phys. 67, 5194 (1990); 10.1063/1.344659

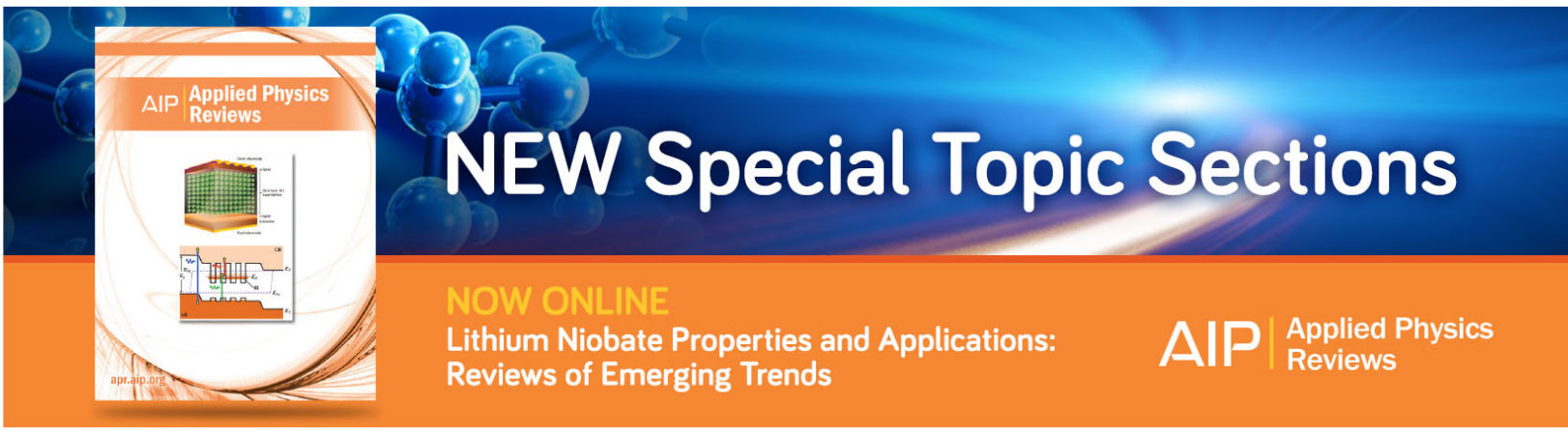




\section{Trends of hybridization-induced magnetism in cerium monochalcogenides}

N. Kioussis and D. Swearingen

Department of Physics, California State University Northridge, Northridge, California 91330

B. R. Cooper

Department of Physics, West Virginia University, Morgantown, West Virginia 26506

J. M. Wills

Los Alamos National Laboratory, Los Alamos, New Mexico 87545

A synthesis of $a b$ initio linear-muffin-tin-orbital (LMTO) electronic structure calculations and a phenomenological model of orbitally driven magnetic ordering has been applied to investigate trends of the effect of hybridization of moderately delocalized $f$ electrons with band electrons on the diverse magnetic behavior across the cerium monochalcogenide series. The parameters entering the Anderson lattice model Hamiltonian are determined from total-energy supercell warped-muffin-tin LMTO calculations with zero, one, and two electrons in the cerium $4 f$ core state. The origins, in the clcctronic structure, of the variation of the density of states at the Fermi energy, the $f$-state resonance width, the hybridization potential, the hybridization-dressed crystal-field splitting, and the hybridization-mediated exchange interactions with the chemical environment (anion size) on going down the chalcogen column have been investigated systematically, increasing thus the degree of $f$-electron localization as the cerium-cerium separation increases.

\section{INTRODUCTION}

Among the cerium compounds, the cerium monopnictides $\mathrm{Ce} X \quad(X=\mathrm{P}, \mathrm{As}, \mathrm{Sb}, \mathrm{Bi})$ and monochalcogenides $(X=\mathrm{S}, \mathrm{Se}, \mathrm{Te})$ provide a class of anomalous compounds exhibiting highly unusual magnetic properties. ${ }^{1,2}$ One of the central features of the anomalous behavior of this class of systems is the presence of an extremcly strong anisotropy even though these systems are cubic of rack-salt structure. The magnetic structures consist of ferromagnetic sheets stacked in various (often complex) arrays, with the cerium magnetic moment aligned perpendicular to the sheets. Neutron-scattering experiments have shown that the magnetic anisotropy changes from the $\langle 001\rangle$ direction in cerium monopnictides ${ }^{1}$ [stacking of ferromagnetic (001) planes] to the $\langle 111\rangle$ direction in cerium monochalcogenides [stacking of (111) planes]. ${ }^{2}$

The crystal-field (CF) splitting of the $4 f$-state manifold is greatly reduced from the value expected by smooth extrapolation of the heavier isostructural rare-earth monopnictides and monochalcogenides. ${ }^{3}$ In both series the CF splitting is about the same, ${ }^{1,2}$ a rather surprising result in view of the additional valence electron on the chalcogen ion, and the CF splitting decreases. with increasing anion size. The magnetic ordering temperature in the pnictide series ${ }^{1}$ increases with increasing anion size from about $8 \mathrm{~K}$ in $\mathrm{CeP}$ to about $25 \mathrm{~K}$ in $\mathrm{CeBi}$, just the contrary to what is observed for the chalcogenides, ${ }^{2}$ where the Néel temperature decreases from about $8 \mathrm{~K}$ in CeS to about $2 \mathrm{~K}$ in $\mathrm{CeTe}$. These unique properties are very sensitive to any perturbation such as pressure, ${ }^{4}$ the dilution of cerium by nonmagnetic $\mathrm{Y}$ or $\mathrm{La},{ }^{5}$ or the substitution of one pnictogen by another pnictogen or chalcogen, ${ }^{4}$ indicating a strong competition between hybridization-mediated exchange interactions and CF effects. While the importance of the band- $f$ hybridization in the diverse magnetic behavior of this class of systems has been generally accepted, ${ }^{1-5}$ it is only recently that systematic calculations ${ }^{6-10}$ have been carried out to examine quantitatively the hybridizationinduced magnetism in the transitional regime between localized and itinerant $f$-electron behavior.

The purpose of this paper is to investigate the origins, in the electronic structure, of the variation of the hybridization-induced magnetism with chemical environment on going down the chalcogen column, increasing thus the degree of $f$-electron localization as the cerium-cerium separation increases. Furthermore, these calculations, which are complementary to our recent calculations, ${ }^{9,10}$ will elucidate the role of the hybridization-induced exchange interactions on the dramatic change in magnetic behavior ongoing from the weakly hybridizing monopnictides to the more strongly hybridizing monochalcogenides of cerium. We have employed a unificd thcory that we have recently developed, which involves a synthesis of (i) a phenomenological theory ${ }^{6-8}$ of orbitally driven magnetic ordering of moderately delocalized $f$-electron systems based on the Anderson lattice Hamiltonian, and (ii) ab initio electronic structure calculations,, 90 based on the linearmuffin-tin-orbital (LMTO) method, ${ }^{11}$ allowing a firstprinciples evaluation of the parameters entering the phenomenological theory.

As the formal aspects of the theory have been reviewed in previous work, ${ }^{6-10}$ we shall forgo all but a brief description of this method here. The self-consistent one-electron potential is obtained ${ }^{9}$ from a warped-muffin-tin LMTO calculation without recourse to the atomic sphere approximation, ${ }^{11}$ i.e., using a nonzero tail parameter as a variational parameter. The full interstitial potential is used and the only shape approximation to the potential is a spherical averaging in nonoverlapping muffin-tin spheres. The cerium $f$ electrons are treated as localized rather than itinerant states and thus they are included self-consistently as 


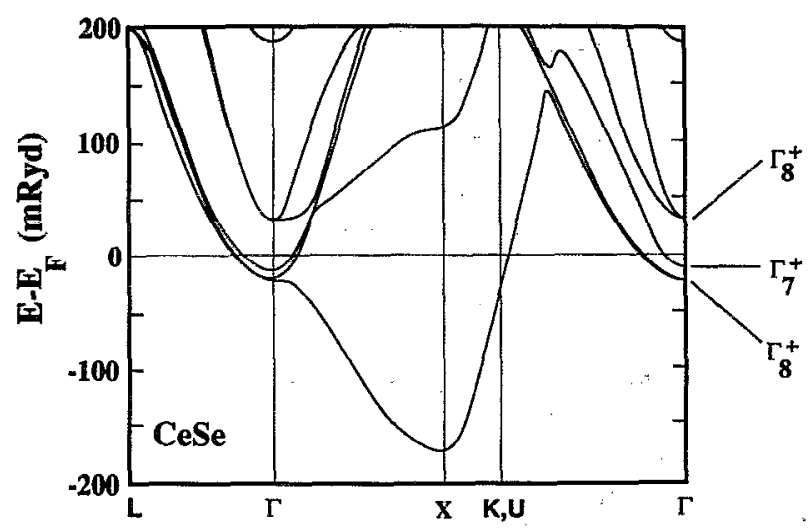

FIG. 1. The band structure of CeSe, calculated with the Ce $4 f$ states treated as core states, along symmetry lines in the Brillouin zone. The band energies are relative to the Fermi energy. The energy bands at $\Gamma$ are Ce $5 d$-derived bands of $\Gamma_{7}^{(+)}$and $\Gamma_{8}^{(+)}$symmetry.

core states at each iteration, not being allowed to hybridize with band states. The relative magnitudes of the muffin-tin radii are chosen so that nearest-neighbor muffin-tin spheres touch at the minimum in the charge density between nearest neighbors. While the basis functions for the band states are scalar relativistic, ${ }^{12}$ spin-orbit coupling is included selfconsistently. The electronic structure calculation provides the band states and energies, and a self-consistent hybridization potential, thereby allowing determination of the hybridization potential matrix elements. The $4 f$-state energy relative to the Fermi energy $E_{f}-E_{F}$, and the intra-atomic correlation energy $U$, are determined from supercell calculations, ${ }^{13}$ treating one cerium ion out of four as a defect differing from the nominal configuration by the addition or subtraction of an electron. Having determined the phenomenological model Hamiltonian parameters on an absolute basis one can evaluate two quantities of interest $t^{6-10}$ : (i) the hybridization dressing (shift) of the crystal-field splitting, and (ii) the hybridization-mediated two-ion exchange interactions, responsible for the unusual magnetic ordering in this class of systems.

\section{RESULTS AND DISCUSSION}

The band structure of CeSe is plotted along symmetry lines in Fig. 1, with the band energies measured with respect to the Fermi energy. At $\Gamma$, the Ce $d$-derived bands are the bases for three representations, one with $\Gamma_{7}^{(+)}$and two with $\Gamma_{8}^{(+)}$symmetry. The addition of an anion $p$ electron on going from the monopnictides to the monochalcogenides of cerium causes the $p$-derived bands to sink far below $E_{F}$, so that the bands dominating the hybridizationinduced properties in the chalcogenide series are largely derived from the $\mathrm{Ce} 5 d$ states. The total density of states (DOS) corresponding to the band structure in Fig. 1 is shown in Fig. 2 (solid curve). Also plotted in the same figure is the partial density of states of the $\mathrm{Ce} d$-derived bands (dotted curve). In all the $\mathrm{Ce}$ monochalcogenides the Fermi energy lies in a high-density-of-states energy region, in contrast to the $\mathrm{Ce}$ monopnictides in which the Fermi

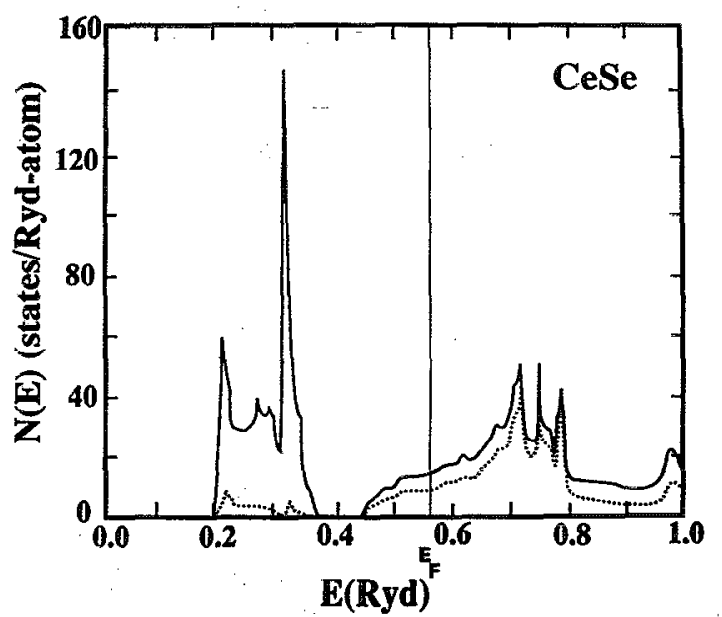

FIG. 2. Total density of states (solid curve) of CeSe corresponding to the band structure in Fig. 1. Also shown is the cerium $d$-derived partial DOS (dotted curve).

energy lies ${ }^{9}$ in a gap between bands of predominantly pnictogen $p$ character and $\mathrm{Ce} d$ character, respectively.

We discuss next the use of our procedure to analyze the source, in the electronic structure of this class of systems, of trends between materials in hybridization-induced effects, by considering the behavior of the two quantities: (i) the calculated $f$-state resonance width $\Gamma$, characterizing the strength of hybridization, ${ }^{6,9,10}$ and (ii) the density of states at the Fermi energy $N\left(E_{F}\right)$, characterizing the number and the character ( $\mathrm{Ce} d$, anion $p$ ) of band states available for hybridization. Calculated values of these quantities are listed in Table I. Also listed in Table I are values of the Fermi energy, the $4 f$ resonant energy $E_{f}$, the partial Ce $d$ - and chalcogen $p$-derived density of states at $E_{F}$, the $4 f$ wave function $\phi_{f}(s)$ at the Ce muffin-tin radius, and the hybridization potential $v(\kappa){ }^{9,10}$ As the degree of $f$-electron localization increases $\left[\phi_{f}(s)\right.$ decreases] on going down the chalcogen column, the resonance width decreases and consequently the strength of $f$-band hybridiza-

TABLE I. Calculated values of the Fermi energy $E_{f}$, the $4 f$ resonant energy $E_{f}$, the total DOS at $E_{F}$, the Ce $d$-and chalcogen $p$-derived partial DOS at $E_{F}$, respectively, the $4 f$ wavefunction $\phi_{f}(s)$ at the Ce muffin-tin radius, the $\mathrm{Ce} 4 f$ resonance width $\Gamma$, and the hybridization potential $v\left(\kappa_{F}\right)$, for the cerium monochalcogenides. Also tabulated are values of the lattice constant $a$ and the Ce muffin-tin radius $s$ in atomic units, respectively.

\begin{tabular}{lccc}
\hline \hline & CeS & CeSe & CeTe \\
\hline$a$ (a.u.) & 10.920 & 11.323 & 12.020 \\
$s$ (a.u.) & 2.7011 & 2.7630 & 2.8367 \\
$E_{F}(\mathrm{Ry})$ & 0.579 & 0.546 & 0.482 \\
$E_{f}(\mathrm{Ry})$ & 0.538 & 0.489 & 0.409 \\
$N\left(E_{F}\right)\left(\mathrm{Ry}^{-1}\right)$ & 12.7 & 14.3 & 15.4 \\
$N_{d}\left(E_{F}\right)\left(\mathrm{Ry}^{-1}\right)$ & 7.9 & 9.4 & 10.3 \\
$N_{p}\left(E_{F}\right)\left(\mathrm{Ry}^{-1}\right)$ & 2.1 & 2.3 & 2.1 \\
$\phi_{f}(s)$ & 0.081 & 0.075 & 0.068 \\
$\Gamma(\mathrm{mRy})$ & 9.72 & 8.33 & 5.67 \\
$v\left(\kappa_{F}\right)(\mathrm{mRy})$ & -4.40 & -3.92 & -3.08 \\
\hline \hline
\end{tabular}


tion; a similar, but smaller in size, trend occurs in the $\mathrm{Ce}$ monopnictides on moving down the column from $\mathrm{CeP}$ to $\mathrm{CeSb}^{6,9}$ The increase of hybridization on going up the chalcogen or pnictogen column has a simple explanation in increased $f$ hybridization with $5 d$ electrons on other cerium sites as the cerium-cerium separation decreases with decreasing anion size. Furthermore, the band- $f$ hybridization, as measured by $\Gamma$, increases by about a factor of 2 on moving from the monopnictides ${ }^{6}$ to the monochalcogenides of cerium. The density of states at $E_{F}, N\left(E_{F}\right)$, which are largely derived from $\mathrm{Ce} 5 d$ states in the chalcogenide series, decreases by about $13 \%$ from $\mathrm{CeS}$ to $\mathrm{CeTe}$. In the monopnictide series, $N\left(E_{F}\right)$, which are derived from both $\mathrm{Ce} 5 d$ and pnictogen $p$ states, are by a factor of 2-3 smaller than the corresponding $N\left(E_{F}\right)$ in the chalcogenide series.

The energy necessary to place the $\mathrm{Ce} f$-electron in a band state at the Fermi energy, $E_{F}-E_{f}$, and the energy required to change from an $f^{1}$ configuration to an $f^{2}$ configuration, $E_{f}+U-E_{F}$, have becn cvaluated for the Ce monochalcogenides from self-consistent supercell calculations. The values of $E_{F}-E_{f}$ and $U$ are then obtained from the $f^{0}, f^{1}$, and $f^{2} f$-state eigenvalues by the use of linear transition theory. ${ }^{13}$ We find that $E_{F}-E_{f}$ and $U$ are 3.7 and $6.2 \mathrm{eV}$ in CeS, 3.8 and $6.0 \mathrm{eV}$ in CeSe, and 3.0 and 6.3 $\mathrm{eV}$ in CeTe, respectively. We have also evaluated the hybridization-induced shift of the "bare" point-charge crystal-field splitting, ${ }^{6-10}$ i.e., $\delta \Delta_{\mathrm{CF}}=\delta E\left(\Gamma_{8}\right)-\delta E\left(\Gamma_{7}\right)$. (The experimentally observed hybridization-dressed crystal-field splitting is $\Delta_{\mathrm{CF}}^{*}=\Delta_{\mathrm{CF}}+\delta \Delta_{\mathrm{CF}}$ ). We find that $\delta \Delta_{\mathrm{CF}}$ decreases from $101 \mathrm{~K}$ in CeTe, to $41 \mathrm{~K}$ in CeSe, to 10 $\mathrm{K}$ in $\mathrm{CeS}$, respectively. In all the chalcogenides, the positive (negative) contributions to $\delta E\left(\Gamma_{7}\right)$ and $\delta E\left(\Gamma_{8}\right)$ resulting from hybridization of band states below (above) $E_{F}$ with the $f^{2}\left(f^{1}\right)$ state are of comparable magnitude, resulting in a small suppression of the crystal-field splitting. In the absence of values for the "bare" crystal-field splitting in this class of systems, it is rather difficult to make a direct comparison of theory with experiment ${ }^{2}$ as in the case of the monopnictide series, where the agreement was found to be excellent. ${ }^{9}$ Including the effects of the full anisotropy of the potential within the muffin-tin spheres, which is currently under investigation, will allow the de- termination of the "bare" crystal-field splitting, and hence will permit a direct comparison with experiment. Work currently in progress is aimed at the evaluation of the hybridization-mediated two-ion exchange interaction parameters, and their effect on the magnetic ordering in this class of systems.

\section{ACKNOWLEDGMENTS}

The research at California State University Northridge (CSUN) was supported through the National. Science Foundation under Grant No. DMR-89-18887, the Research Corporation under Grant No. C-2707, and the Office of Research and Sponsored Projects at CSUN. The research at West Virginia University was supported through the National Science Foundation under Grant No. DMR-88-07523, and at Los Alamos National Laboratory by the U.S. Department of Energy.

${ }^{1}$ J. Rossat-Mignod, P. Burlet, S. Quezel, J. M. Effantin, D. Delacote, H. Bartholin, O. Vogt, and D. Ravot, J. Magn. Magn. Mater. 31-34, 398 (1983).

${ }^{2}$ H. R. Ott, J. K. Kjems, and F. Hulliger, Phys. Rev. Lett. 42, 1378 (1979); F. Hulliger, B. Natterer, and H. R. Ott, J. Magn. Magn. Mater. 8, 87 (1978)

${ }^{3}$ R. J. Birgeneau, E. Bucher, J. P. Maita, L. Passell, and K. C. Turberfield, Phys. Rev. B 8, 5345 (1973).

${ }^{4}$ J. Rossat-Mignod, J. M. Effantin, P. Burlet, T. Chattopadhyay, L. P. Regnault, H. Bartholin, C. Vettier, O. Vogt, D. Ravot, and J. C. Achart, J. Magn. Magn. Mater. 52, 111 (1985).

${ }^{5}$ B. R. Cooper and O. Vogt, J. Phys. (Paris) Colloq. 32, C1-1026 (1971).

${ }^{6}$ B. R. Cooper, J. M. Wills, N. Kioussis, and Q.-G. Sheng, J. Phys. (Paris) Colloq. C8-49, 463 (1988); B. R. Cooper, J. M. Wills, N. Kioussis, and Q.-G. Sheng, J. Appl. Phys. 64, 5587 (1988).

${ }^{7}$ B. R. Cooper, R. Siemann, D. Yang, P. Thayamballi, and A. Banerjea, in Handbook on the Physics and Chemistry of the Actinides, edited by A. J. Freeman and G. H. Lander (North-Holland, Amsterdam, 1985), Vol. 2, Chap. 6, pp. 435-500.

${ }^{8}$ N. Kioussis, B. R. Cooper, and A. Banerjea, Phys. Rev. B 38, 9132 (1988).

${ }^{9}$ J. M. Wills and B. R. Cooper, Phys. Rev. B 36, 3809 (1987).

${ }^{10}$ N. Kioussis, B. R. Cooper, J. M. Wills, and Q.-G. Sheng, Physica 163, 107 (1990); N. Kioussis, B. R. Cooper, and J. M. Wills, J. Appl. Phys. 63, 3683 (1988).

${ }^{11}$ O. K. Andrersen, Phys. Rev. B 12, 3060 (1975).

${ }^{12}$ D. D. Koelling and B. N. Harmon, J. Phys. C 10, 3107 (1977).

${ }^{13}$ M. R. Norman, D. D. Koelling, A. J. Freeman, H. J. F. Jansen, B. I. Min, T. Oguchi, and L. Ye, Phys. Rev. Lett. 53, 1673 (1984) 